\title{
Stimulated Reporting: The Impact of US Food and Drug Administration-Issued Alerts on the Adverse Event Reporting System (FAERS)
}

\author{
Keith B. Hoffman • Andrea R. Demakas • \\ Mo Dimbil · Nicholas P. Tatonetti • \\ Colin B. Erdman
}

Published online: 26 September 2014

(C) The Author(s) 2014. This article is published with open access at Springerlink.com

\begin{abstract}
Background The US Food and Drug Administration (FDA) uses the Adverse Event Reporting System (FAERS) to support post-marketing safety surveillance programs. Currently, almost one million case reports are submitted to FAERS each year, making it a vast repository of drug safety information. Sometimes cited as a limitation of FAERS, however, is the assumption that "stimulated reporting" of adverse events (AEs) occurs in response to warnings, alerts, and label changes that are issued by the FDA.

Objective To determine the extent of "stimulated reporting" in the modern-day FAERS database.

Methods One hundred drugs approved by the FDA between 2001 and 2010 were included in this analysis. FDA alerts were obtained by a comprehensive search of the FDA's MedWatch and main websites. Publicly available FAERS data were used to assess the "primary suspect" AE reporting pattern for up to four quarters before, and after, the issuance of an FDA alert.

Results A few drugs did demonstrate "stimulated reporting" trends. A majority of the drugs, however,
\end{abstract}

Electronic supplementary material The online version of this article (doi:10.1007/s40264-014-0225-0) contains supplementary material, which is available to authorized users.

K. B. Hoffman $(\bowtie) \cdot$ A. R. Demakas · M. Dimbil ·

C. B. Erdman

AdverseEvents, Inc., 3663 N. Laughlin Road,

Suite 102, Santa Rosa, CA 95403, USA

e-mail: keith@adverseevents.com

N. P. Tatonetti

Department of Biomedical Informatics, Columbia University,

New York, USA showed little evidence for significant reporting changes associated with the issuance of alerts. When we compared the percentage changes in reporting after an FDA alert with those after a sham "control alert", the overall reporting trends appeared to be quite similar. Of 100 drugs analyzed for short-term reporting trends, 21 real alerts and 25 sham alerts demonstrated an increase (greater than or equal to $1 \%$ ) in reporting. The long-term analysis of 91 drugs showed that 24 real alerts and 28 sham alerts demonstrated a greater than or equal to $1 \%$ increase.

Conclusions Our results suggest that most of modern day FAERS reporting is not significantly affected by the issuance of FDA alerts.

\section{Key Points}

Adverse event (AE) databases such as the US Food and Drug Administration (FDA) Adverse Event Reporting System are assumed to suffer from "stimulated reporting".

"Stimulated reporting" is the concept that public disclosure of a safety issue by the issuance of, for example, an FDA alert will result in substantially increased AE rates for the drug and/or specific safety issue mentioned in such an alert.

We analyzed both overall and AE-specific reporting before and after 100 FDA alerts and found no discernable pattern of increased reporting.

While certain drugs appeared to have their $\mathrm{AE}$ reporting trends affected by an FDA warning, most did not. 


\section{Introduction}

Because of financial and logistical hurdles, pre-approval clinical trials can never be large enough, or long enough, to identify and properly characterize all adverse effects that may occur once a drug is introduced to consumer populations. The gradual evolution of adverse event (AE) profiles across numerous drugs (e.g., thalidomide, sibutramine, cerivastatin, rofecoxib) [1-4], after they were approved by the US Food and Drug Administration (FDA), serves to underscore the preceding points.

Unfortunately, the time lag associated with the dissemination of relevant post-marketing $\mathrm{AE}$ information is also a significant concern. In fact, within 7 years after FDA approval, only half of a drug's serious post-marketing AEs was listed in the Physician's Desk Reference, a main source of AE information for many prescribers [5]. Such delays, combined with the aforementioned limitations of the pre-approval clinical trial process, reinforce the need for diligent post-marketing vigilance [5-8].

The FDA's Adverse Event Reporting System (FAERS) [9] is a centralized computerized information database that is broadly used by the agency, and other pharmacovigilance experts, for post-marketing drug safety surveillance $[6,8,10]$. International government and related organizations also use spontaneous AE databases to identify postapproval drug safety concerns.

Challenges to using FAERS data, however, have been reported to include under-reporting [11, 12], the "Weber Effect" [13, 14], and "stimulated reporting" [15-19]. With regard to under-reporting, while it remains a significant limitation, recent efforts by the FDA, global regulatory agencies, and the healthcare industry itself are helping to increase $\mathrm{AE}$ reporting rates. Indeed, almost one million $\mathrm{AE}$ reports will be added to both the EudraVigilance [20] and FAERS databases this year alone [21]. FAERS now has a total of over seven million reports. With regard to the "Weber effect", a recent FAERS study [22] demonstrated that it may be of less concern than it was in the past, likely owing to an increasing focus on the importance and utility of post-approval AE reporting by both regulatory and key healthcare players $[22,23]$. Unfortunately, organized FAERS data are not readily accessible to healthcare professionals who instead rely heavily on safety information from drug label "inserts" that are often based predominantly on pre-approval clinical trial results and are frequently inadequate with regard to AE details [24].

To help guide prescribing decisions and arm healthcare professionals with emerging post-marketing safety risks, the FDA issues warnings [7, 18, 25], which have often effectively modified prescribing behaviors $[17,19]$. However, the alerts are sometimes challenged by the scientific community [17, 26-29], numerous alerts can be required to trigger an effect [30], and too often they have little to no impact on prescribing patterns [17, 28, 31-33]. Occasionally, regulatory action by the FDA can also have the unintended consequence of depriving patients of efficacious and safe (when actually used as directed) medications [34-39].

"Stimulated reporting" refers to the concept that public disclosure of a safety issue by the issuance of, for example, an FDA alert will result in substantially increased AE reporting rates regarding the drug and/or the specific $\mathrm{AE}$ mentioned in such an alert. The term can also refer to clustering of $\mathrm{AE}$ reports triggered by the activities of consumer-based "support groups" and/or reporting activity related to litigation. Stimulated reporting has important implications regarding the utility of post-marketing $\mathrm{AE}$ data, as alert-driven shifts in reporting could impact the accuracy of comparative research and related analytical methods such as disproportionality analysis.

Although a number of studies have evaluated the impact of FDA alerts on drug use [16-19, 31, 33, 40-57], few have comprehensively studied the impact of FDA alerts on AE reporting. Past studies that have found evidence for stimulated reporting with regard to post-marketing $\mathrm{AE}$ data have generally examined solitary, or a single group of, drugs.

A 1998-2004 study showed that the reporting frequency of key AEs associated with statin use increased as a result of FDA warnings, but a general increase in broad AE reporting for drugs in the class was not observed [58]. A study of Italian AE databases indicated that three out of four regulatory warnings triggered increased $\mathrm{AE}$ reporting [38]. FDA-issued boxed warnings were linked to increases in FAERS reporting for multiple myeloma drugs [59]. Two French studies found evidence for stimulated reporting via changes in disproportionality [60, 61]. A study from The Netherlands found a media influence regarding cardiac arrhythmias linked to antihistamines [62]. An Australian study found an increase in reporting odds ratios after media publicity regarding links between a sleep medication and parasomnias and amnesia [63]. An FDA alert influenced FAERS reporting of pancreatitis linked to two diabetic medications [64]. FDA researchers detailed a large increase in rhabdomyolysis reports linked to statin drugs after an alert was issued on cerivastatin [65].

While select FDA alerts appeared to have impacted AE reporting rates, we have not found a broad study regarding FAERS reporting trends after alert(s) are issued. Therefore, we used a big data analytic platform [66] to quantify FAERS reporting trends over a 10 -year period regarding 100 drugs that had been issued an FDA alert. 


\section{Methods}

\subsection{FAERS Data Preparation}

Case reports that were missing or contained malformed key identification fields [Individual Safety Report number (ISR), patient number, drug sequence identification, or Medical Dictionary for Regulatory Activities (MedDRA ${ }^{\circledR}$ ) AE term] were discarded. As long as the aforementioned key identification fields were contained in a given case report, allowable missing fields included: age, gender, weight, outcome, and condition. Cases were discarded if the drug name was found to be indeterminate or if the name was determined to not represent an FDA-approved drug (e.g. dietary supplements, foods). In an effort to exclude pre-approval AE case reports mistakenly logged into the FAERS, the date of receipt for a given case report must have occurred after the drug's FDA approval date.

In instances where there were multiple, individual case safety reports for the same identification number, we selected the earliest reported case. For example, if a patient reported adverse effects from the same drug in 2008 and 2010 and we ran a search against all primary suspect cases for that drug across all dates, only the 2008 report would be included. However, if we were to run a search against all primary suspect cases for 2010 only, then the 2010 case would be included. Because the case counts included in our analysis were restricted by quarter, drug, and, when applicable, AE, we are confident that we obtained a complete profile of the primary suspect cases of interest.

Drug name text-mapping was accomplished as previously described by Hoffman et al. [66]. Drug names were normalized to RxNorm reference codes [67] using string searching and manual curation. National Drug File Reference Terminology [68] was used to provide ancillary information on class and mechanism of action.

AE information was coded according to MedDRA ${ }^{\circledR}$ version 16.1 [69]. AEs mentioned in each safety alert (see Electronic Supplemental Material) were mapped to MedDRA terms according to the following hierarchy: (1) AEs linked to specific medical conditions were coded verbatim to MedDRA Preferred Terms (i.e., "retinal detachment" was mapped to the MedDRA Preferred Term AE "retinal detachment"); (2) variations of specific AEs were also coded to MedDRA Preferred Terms (i.e., "heart attack" was mapped to the Preferred Term "myocardial infarction"); (3) AEs highlighting disorders pertaining to specific organs or parts of the body were coded to MedDRA System Organ Classes (i.e., "Central Nervous System Disorders" was mapped to the System Organ Class "Nervous system disorders"); and (4) generalized alert terms that encompassed multiple specific AEs were coded to MedDRA
Standardized MedDRA ${ }^{\circledR}$ Queries (SMQs) (i.e., "hypertension" was mapped to all Preferred Terms that fall under the SMQ "Hypertension").

"Primary suspect" designations in FAERS case reports were quantified in an attempt to restrict the analysis to those drugs directly suspected of causing the AE. ("Primary suspect" is a description chosen by the person who submitted a given case report and is their estimate of which drug, if the subject was taking more than one, was likely responsible for the observed $\mathrm{AE}$ ).

Finally, drugs were mapped to their corresponding Anatomical Therapeutic Chemical (ATC) codes [70] in an attempt to determine if $\mathrm{AE}$ reporting patterns differed across therapeutic class (see Electronic Supplemental Material).

\subsection{FDA Alert Data Collection}

We searched for FDA alerts, and their associated issuance dates, via the FDA's MedWatch [71] and main [72] websites.

A data mining platform specifically built to optimize FAERS data [66] was used to quantify "primary suspect" case counts up to four calendar quarters before, and four after, publicized FDA regulatory actions (Dear Healthcare Professional Letter, Drug Safety Communication, Early Communication, FDA Information for Healthcare Professionals, FDA Recall, Public Health Advisory, REMS Issued, and Safety Labeling Changes).

\subsection{Statistical Analysis}

For our primary analysis, the number of quarterly AE cases was collected for each drug for 2000-2012. Percentage differences were obtained for quarter two and quarter four prior, vs. quarter two and quarter four after, an FDA or a sham alert and displayed as a histogram. Percentiles were binned into 25 or $50 \mathrm{U}$ increments starting with a minimum bin of " -99 to -75 " and ending at a maximum "1,200-1,249" bin.

Prescription drugs approved from 2000 to 2010 were eligible for analysis. We assessed changes in short-term reporting by comparing the amount of total reports in the two quarters prior to the quarter the FDA alert was issued in with the two quarters following such an alert. The same method was used to assess longer-term reporting trends by comparing four quarters before with four quarters after the same FDA alert. Our inclusion criteria were: (1) two or four full quarters between the FDA-approval date and alert date for all two quarter and four quarter calculations, respectively, (2) a minimum of four quarters was needed between the alert date and quarter four 2012, (3) the drug needed to have a minimum of 40 primary suspect cases in 
the both the alert quarter and the quarter directly prior to the alert for the "all primary suspect" case report analysis, and (4) the drug needed to have a minimum of five AE/ drug-specific cases in the both the alert quarter and the quarter directly prior to the alert for the "AE-specific" case report analysis. Over-the-counter and "street" drugs, vaccines, and broad, undefined compounds listed in FAERS were not analyzed.

For internal control analysis, we used each drug ("all event analysis") as well as the specific AE/drug pairs as their own controls by assigning a "sham alert" five quarters before the actual FDA alert. We then evaluated the percentage changes in reports (for both two quarter and four quarter differences) for the sham alert in the same manner that we analyzed drugs with actual FDA alerts. We used the Mann-Whitney test to determine if there were statistical differences between reporting rates for real compared with sham alerts.

\section{Results}

Figure 1 shows both the short- and long-term percent changes in primary case reporting for drugs that were subjected to an FDA alert during the time period studied. One hundred drugs were analyzed for short-term (two quarters before and after alerts) effects, while 91 of those 100 drugs were analyzed for long-term (four quarters before and after alerts) effects.

Figure 2 shows a short-term (two quarters prior vs. two quarters after) comparison of reporting changes for 43 out of 100 drugs that could serve as their own internal controls (by having a sham alert set at five quarters before their actual FDA alert). 21 real alerts and 25 sham alerts demonstrated an increase (greater than or equal to $1 \%$ ) in short-term reporting. If one were to define "stimulated reporting" as a $50 \%$ or higher increase in reporting, then 7 out of 43 actual FDA alerts and 7 out of 43 of sham alerts would qualify.

For Fig. 2, we ran a rank sum (Mann-Whitney) test comparing the two quarters prior vs. two quarters post results for actual alerts compared with sham alerts. The median percent change was not significantly different for actual alerts $(1 \%)$ vs. sham alerts $(9 \%)$ with $U=957$ $(p=0.78)$.

Figure 3 shows a long-term (four quarters prior vs. four quarters after) comparison of reporting changes for 43 out of 100 drugs that could serve as their own internal controls (by having a sham alert set at five quarters before their actual FDA alert). 24 real alerts and 28 sham alerts demonstrated an increase (greater than or equal to $1 \%$ ) in longterm reporting. If one were to define "stimulated reporting" as a $50 \%$ or higher increase in reporting then 12 out of 43 actual FDA alerts and 12 out of 43 sham alerts would qualify.

For Fig. 3, we ran a rank sum (Mann-Whitney) test comparing the four quarters prior vs. four quarters post results for actual alerts compared with sham alerts. The median percent change was not significantly different for actual alerts (5\%) vs. sham alerts $(30 \%)$ with $U=1,022$ $(p=0.40)$.

Figure 4 shows both the short- and long-term percent changes in reporting of specific AE/drug pairs noted in FDA alerts. 134 AE/drug pairs were analyzed for shortterm (two quarters before and after alerts) effects, while 124 of those $134 \mathrm{AE} / \mathrm{drug}$ pairs were analyzed for longterm (four quarters before and after alerts) effects.

Figure 5 shows a short-term (two quarters prior vs. two quarters after) comparison of reporting changes for 61 out of $134 \mathrm{AE} /$ drug pairs that could serve as their own internal controls (by having a sham alert set at five quarters before their actual FDA alert). 26 real alerts and 25 sham alerts demonstrated an increase (greater than or equal to $1 \%$ ) in AE-specific reporting. If one were to define "stimulated

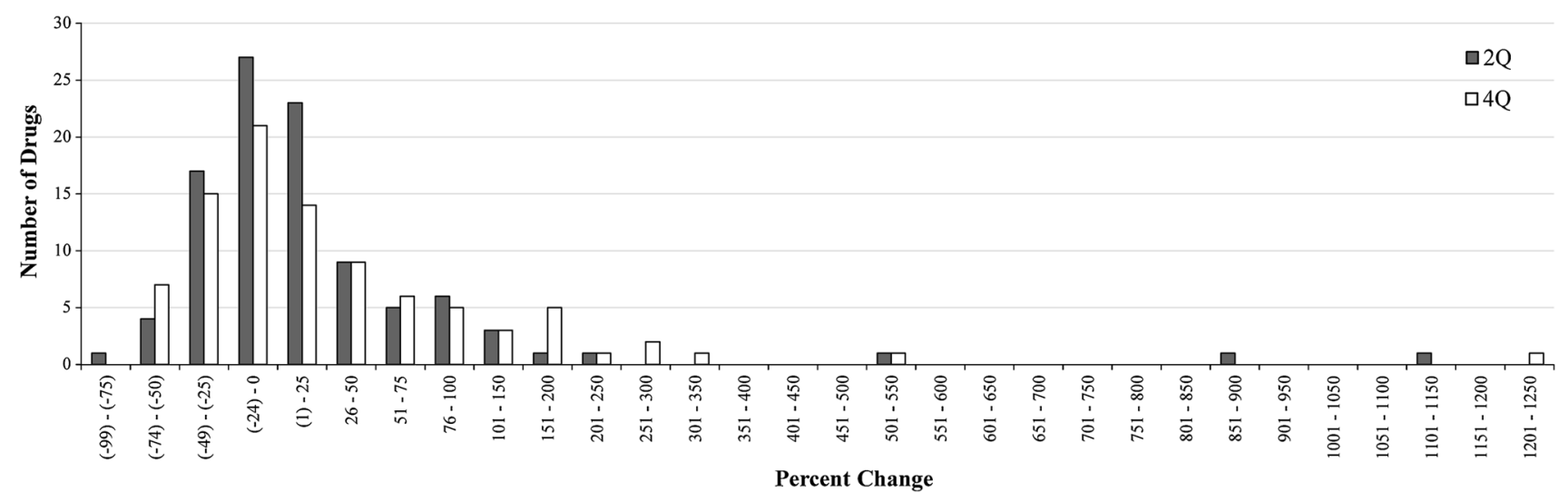

Fig. 1 Short- and long-term percent changes in primary case reporting for drugs that were subjected to a US Food and Drug Administration alert during the time period studied. $2 Q$ two quarters, $4 Q$ four quarters 


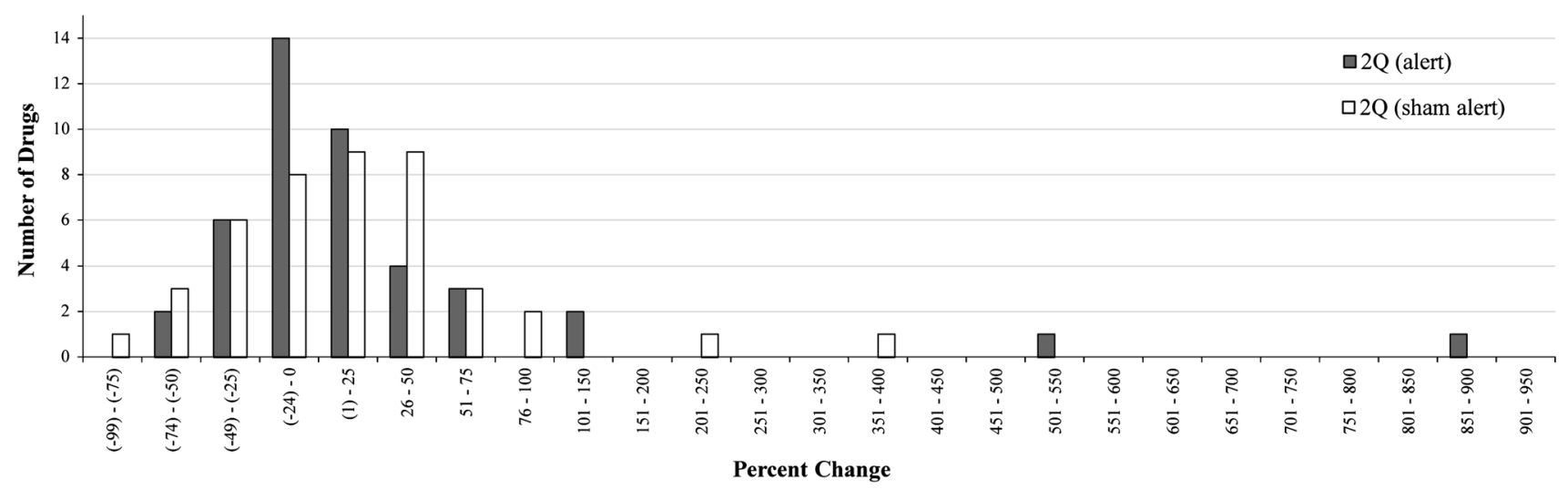

Fig. 2 Short-term percent changes in primary case reporting for real alerts compared with sham alerts. $2 Q$ two quarters

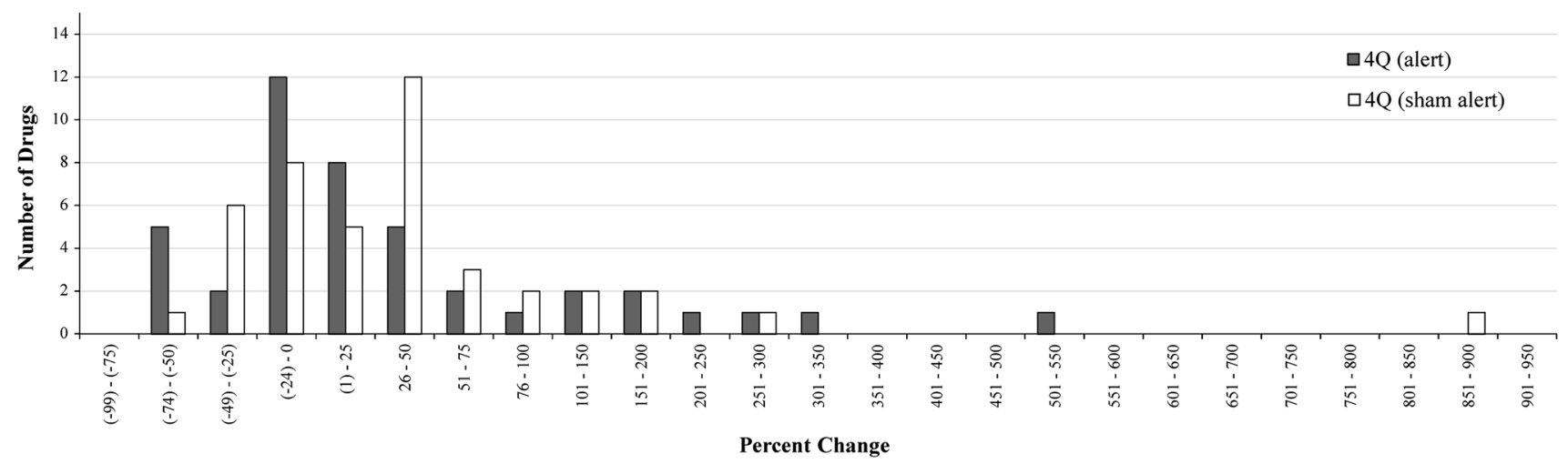

Fig. 3 Long-term percent changes in primary case reporting for real alerts compared with sham alerts. $4 Q$ four quarters

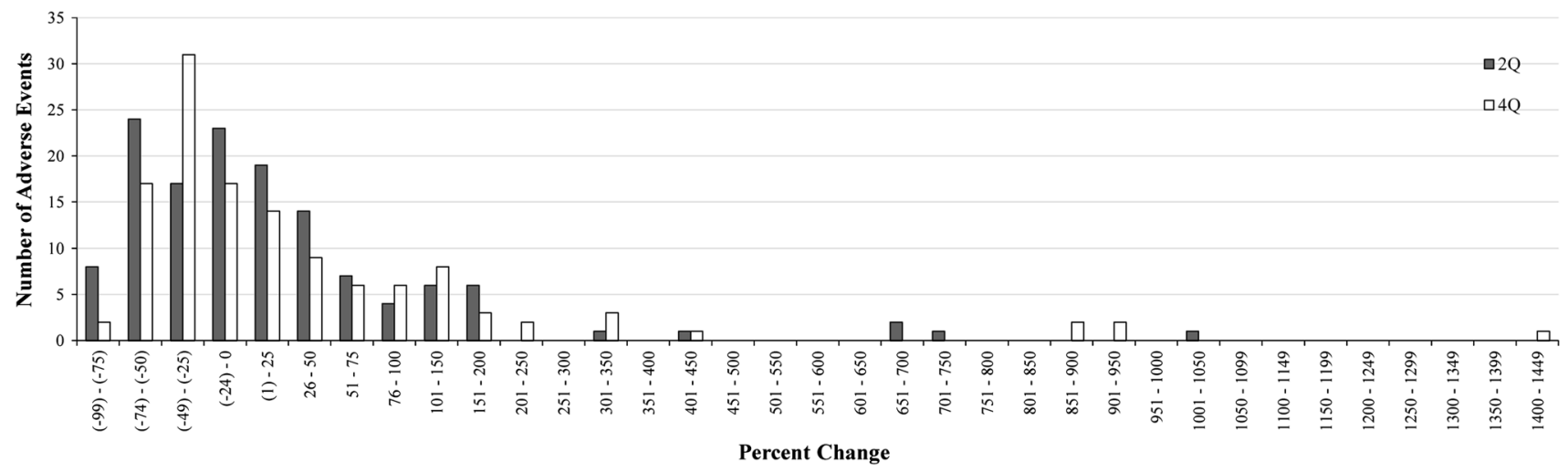

Fig. 4 Short- and long-term percent changes in specific adverse event/drug pair case reporting for drugs that were subjected to an FDA alert during the time period studied. $2 Q$ two quarters, $4 Q$ four quarters

reporting" as a $50 \%$ or higher increase in reporting, then 10 out 61 actual FDA alerts and 14 out of 61 sham alerts would qualify.

For Fig. 5, we ran a rank sum (Mann-Whitney) test comparing the two quarters prior vs. two quarters post results for actual alerts compared with sham alerts. The median percent change was not significantly different for actual alerts $(-3 \%)$ vs. sham alerts $(0 \%)$ with $U=1,968$ $(p=0.47)$.

Figure 6 shows a long-term (four quarters prior vs. four quarters after) comparison of reporting changes for 61 out of $134 \mathrm{AE} /$ drug pairs that could serve as their own internal controls (by having a sham alert set at five quarters before their actual FDA alert). 30 real alerts and 49 sham alerts 


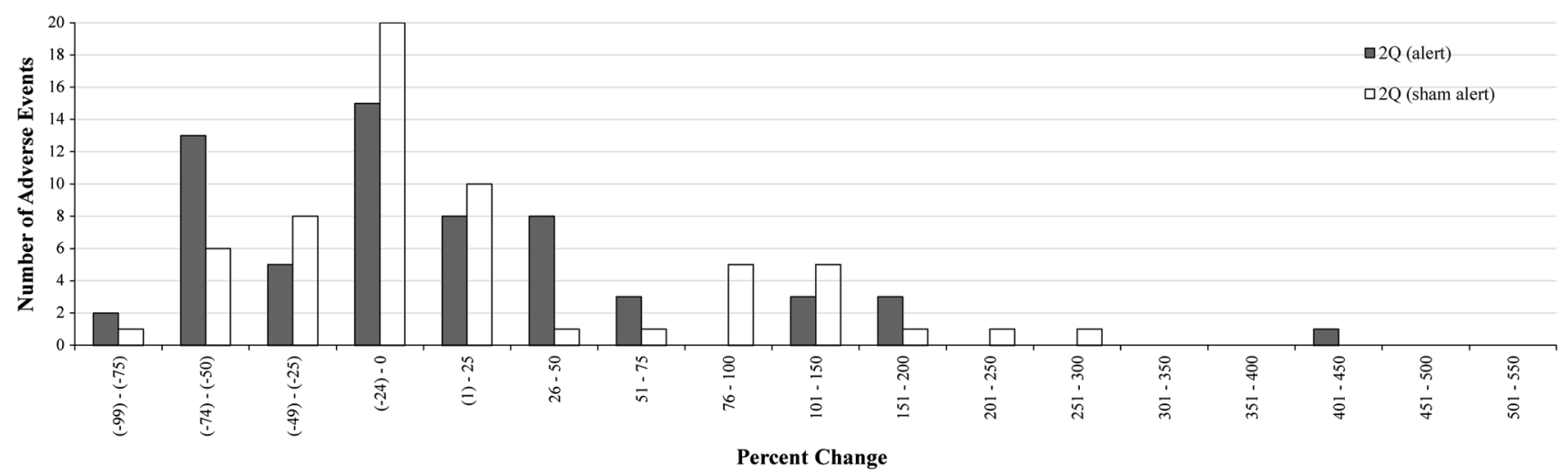

Fig. 5 Short-term percent changes in specific adverse event/drug pair case reporting for real alerts compared with sham alerts. $2 Q$ two quarters

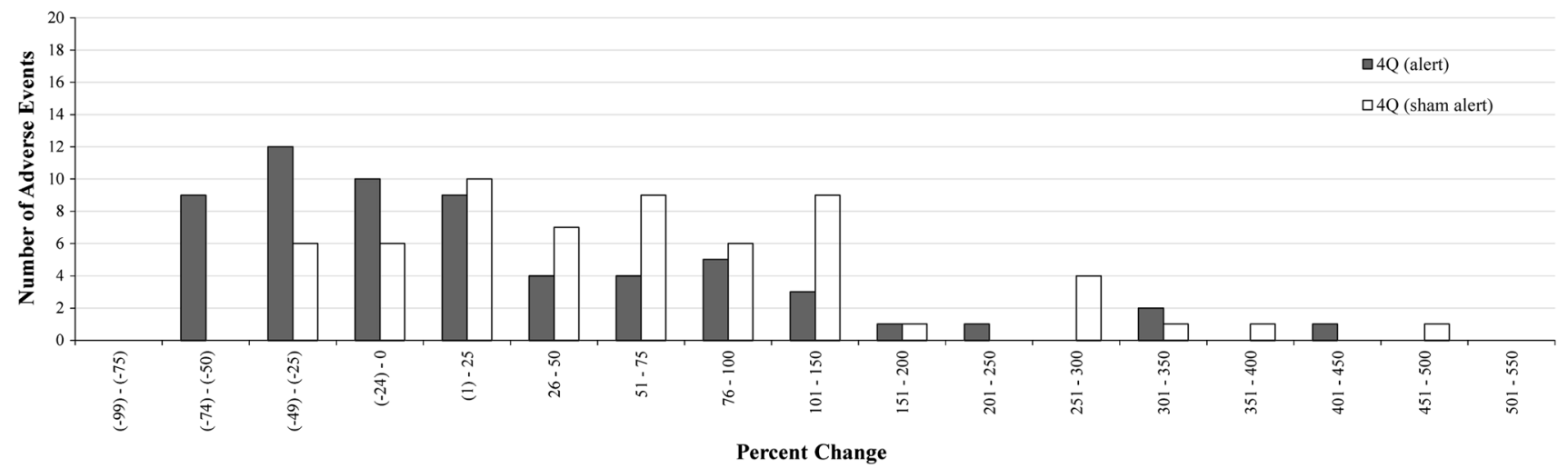

Fig. 6 Long-term percent changes in specific adverse event/drug pair case reporting for real alerts compared with sham alerts. $4 Q$ four quarters

demonstrated an increase (greater than or equal to $1 \%$ ) in AE-specific reporting. If one were to define "stimulated reporting" as a 50\% or higher increase in reporting, then 17 out of actual FDA alerts and 33 out of 61 sham alerts would qualify.

For Fig. 6, we ran a rank sum (Mann-Whitney) test comparing the four quarters prior vs. four quarters post results for actual alerts compared with sham alerts. The median percent change was significantly lower for actual alerts $(-4.8 \%)$ than for sham alerts $(59.3 \%)$ with $U=2,603(p=0.000145)$.

\section{Discussion}

With regard to AE reporting, while we did find evidence for clear cases of "stimulated reporting" in a subset of drugs examined, the majority of FDA alerts did not appear to undergo substantial changes in reporting rates because of such warnings. When we compared $\mathrm{AE}$ reporting trends before and after both FDA alerts and sham alerts, the overall pattern of AE reporting was very similar between the groups. This lack of an overall "stimulated" reporting pattern held for the short-term (two quarters before and after an alert was issued) and long-term (four quarters before and after) analyses, as well as the all primary suspect cases and AE-specific/drug pair analysis.

Numerous factors are likely to influence whether a given FDA alert results in "stimulated" changes in drug use and/or AE reporting, including: how serious the AE mentioned in the alert is, whether the guidance requires increased workload for prescribers, the extent of FDA and media coverage, whether the scientific basis of the warning is widely accepted, the specificity of the warning, and whether the alert includes guidance for the selection of safer alternatives.

Traditional media coverage also shapes the dissemination of emerging drug safety information and can significantly affect the impact of FDA warnings (for a review, see Yong et al. [73]). One study found that $100 \%$ of newspaper articles included at least one mention of a benefit of a newly approved drug, while only $32 \%$ mentioned at least one harmful effect [74]. The Internet can also be a significant factor in behavioral changes to drug safety information [75]. Unfortunately, sometimes the message delivered by regulatory authorities is not the same one that is relayed by the media [73, 76-78]. 
Willingness to follow the FDA's guidance is yet another variable that can have a significant impact. For example, in one study of REMS guidance, $71 \%$ of prescribing physicians stated that it would be "somewhat" to a "very" significant barrier to have to endure a locally available, one-time training session of less than $2 \mathrm{~h}$ to guide them on the proper use of emerging opioids [28]. Along the same lines, another study demonstrated that prescribers are less likely to comply with guidance that entails increased patient contact [49]. Additionally, a specific FDA guidance suggesting "close supervision" of patients to monitor something as serious as increased risk of suicide appeared to be entirely disregarded [48].

As in most areas of risk communication, FDA warnings and alerts appear to be most effective when they are specific, offer alternative options, and are repeated. Indeed, other studies [17, 79-82] have suggested that FDA alerts must be concise, actively publicized through scientific and traditional media, and offer immediate prescribing options (not added educational programs or increased patient time) for warnings to trigger meaningful prescribing changes.

We are encouraged that modern FAERS data do not appear to suffer from the biases that would be introduced by significant shifts in reporting owing to the issuance of FDA alerts.

\subsection{Limitations}

The limitations of our study include the fact that we did not have data to correlate how much media coverage accompanied each FDA warning, nor any way to gauge how well each warning was communicated to, or received by, prescribers. We were not able to determine if any of the FDA alerts we analyzed were pre-dated by alerts issued in nonUS countries. We measured the "primary suspect" case reports in an attempt to restrict the analysis to those drugs directly suspected of causing the $\mathrm{AE}$, but such a selection process could have unintended consequences with regard to the analysis of stimulated reporting as the impact on secondary suspects was not studied. Scrip (drug usage) numbers were not available to correlate with $\mathrm{AE}$ counts. The inclusion criteria of 40 cases were intended to focus the analysis on drugs that had sizeable use rates and was also needed to make the dataset a manageable size. Such steps, however, may have excluded certain drugs where $\mathrm{AE}$ reporting trends went up or down after an alert. Using each drug as its own internal control necessitated the use of drugs that had at least nine quarters of FAERS data before an actual FDA alert, potentially biasing the sham alert analysis towards slightly older drugs. Our results are based upon the FAERS database alone, so the relation between our findings and smaller databases is unknown. The strength of this research is that it comprehensively examined a large number of individual drugs, included all FDA warning types, and analyzed trends over an extended period of time.

\section{Conclusion}

While there is no doubt that occasionally an FDA alert can have a profound influence on $\mathrm{AE}$ reporting rates for a given drug, we were struck by how similar the overall reporting trend distributions were between drugs with real compared with sham alerts. We therefore suggest that modern day $\mathrm{AE}$ reporting trends do not appear to be substantially affected by FDA alerts.

Acknowledgments The research leading to these results was funded entirely by AdverseEvents, Inc., a private corporation.

MedDRA $^{\circledR}$, the Medical Dictionary for Regulatory Activities terminology, is the international medical terminology developed under the auspices of the International Conference on Harmonization of Technical Requirements for Registration of Pharmaceuticals for Human Use.

Keith B. Hoffman, Andrea R. Demakas, Mo Dimbil, and Colin B. Erdman have all declared employment- and stock-related conflicts of interests in their declaration forms related to AdverseEvents, Inc. Nicholas P. Tatonetti has declared a stock-related conflict of interest in his declaration forms related to AdverseEvents, Inc. Keith B. Hoffman, Andrea R. Demakas, Mo Dimbil, Nicholas P. Tatonetti, and Colin B. Erdman have no other conflicts of interest that are directly relevant to the content of this manuscript.

Keith B. Hoffman conceived of the study, analyzed and interpreted the data, and drafted and approved the final submitted manuscript. Nicholas P. Tatonetti made suggestions for data interpretation and approved the final submitted manuscript. Andrea Demakas, Mo Dimbil, and Colin B. Erdman collected data and approved the final submitted manuscript.

Open Access This article is distributed under the terms of the Creative Commons Attribution Noncommercial License which permits any noncommercial use, distribution, and reproduction in any medium, provided the original author(s) and the source are credited.

\section{References}

1. Charatan F. Bayer decides to withdraw cholesterol lowering drug. BMJ. 2001;323(7309):359.

2. FDA. Safety information: Vioxx (rofecoxib). 2002. http://www. fda.gov/Safety/MedWatch/SafetyInformation/SafetyAlertsfor HumanMedicalProducts/ucm154520.htm. Accessed August 2014.

3. FDA. Follow-up to the November 2009 early communication about an ongoing safety review of sibutramine, Marketed as Meridia. 2010. http://www.fda.gov/Drugs/DrugSafety/Postmarket DrugSafetyInformationforPatientsandProviders/DrugSafetyInfor mationforHeathcareProfessionals/ucm198206.htm. Accessed August 2014.

4. Rehman W, Arfons LM, Lazarus HM. The rise, fall and subsequent triumph of thalidomide: lessons learned in drug 
development. Ther Adv Hematol. 2011;2(5):291-308. doi:10. 1177/2040620711413165.

5. Lasser KE, Allen PD, Woolhandler SJ, Himmelstein DU, Wolfe SM, Bor DH. Timing of new black box warnings and withdrawals for prescription medications. JAMA. 2002;287(17):2215-20.

6. Ahmad SR. Adverse drug event monitoring at the Food and Drug Administration. J Gen Intern Med. 2003;18(1):57-60.

7. Lindstrom JA. Sources of drug information: FDA-approved labeling and other official FDA sources. Dermatol Ther. 2009;22(3):246-56. doi:10.1111/j.1529-8019.2009.01238.x.

8. Weaver J, Grenade LL, Kwon H, Avigan M. Finding, evaluating, and managing drug-related risks: approaches taken by the US Food and Drug Administration (FDA). Dermatol Ther. 2009;22(3):204-15. doi:10.1111/j.1529-8019.2009.01233.x.

9. FDA. FDA Adverse Event Reporting System (FAERS) (formerly AERS). 2012. http://www.fda.gov/drugs/guidancecompliance regulatoryinformation/surveillance/adversedrugeffects/default.htm. Accessed August 2014.

10. FDA. Potential signals of serious risks/new safety information identified from the FDA Adverse Event Reporting System (FAERS) (formerly AERS). 2014. http://www.fda.gov/Drugs/Guidance ComplianceRegulatoryInformation/Surveillance/AdverseDrugEffects/ ucm082196.htm. QuarterlyReports. Accessed August 2014.

11. Hazell L, Shakir SA. Under-reporting of adverse drug reactions: a systematic review. Drug Saf Int J Med Toxicol Drug Exp. 2006;29(5):385-96.

12. Lopez-Gonzalez E, Herdeiro MT, Figueiras A. Determinants of under-reporting of adverse drug reactions: a systematic review. Drug Saf Int J Med Toxicol Drug Exp. 2009;32(1):19-31. doi:10. 2165/00002018-200932010-00002.

13. Weber J. Epidemiology of adverse reactions to nonsteroidal antiinflammatory drugs. Adv Inflamm Res. 1984;6:1-7.

14. Weber J. Epidemiology in the United Kingdom of adverse drug reactions from non-steroidal anti-inflammatory drugs. SideEffects of Anti-Inflammatory Drugs Inflammation and Drug Therapy Series 1987.

15. Dasgupta N, Mandl KD, Brownstein JS. Breaking the news or fueling the epidemic? Temporal association between news media report volume and opioid-related mortality. PLoS One. 2009;4(11):e7758. doi:10.1371/journal.pone.0007758.

16. Busch SH, Frank RG, Leslie DL, Martin A, Rosenheck RA, Martin EG, et al. Antidepressants and suicide risk: how did specific information in FDA safety warnings affect treatment patterns? Psychiatr Serv. 2010;61(1):11-6. doi:10.1176/appi.ps.61.1.11.

17. Dusetzina SB, Higashi AS, Dorsey ER, Conti R, Huskamp HA, Zhu S, et al. Impact of FDA drug risk communications on health care utilization and health behaviors: a systematic review. Med Care. 2012;50(6):466-78. doi:10.1097/MLR.0b013e318245a160.

18. Piening S, Haaijer-Ruskamp FM, de Vries JT, van der Elst ME, de Graeff PA, Straus SM, et al. Impact of safety-related regulatory action on clinical practice: a systematic review. Drug Saf Int J Med Toxicol Drug Exp. 2012;35(5):373-85. doi:10.2165/ 11599100-000000000-00000.

19. Conti RM, Dusetzina SB, Herbert AC, Berndt ER, Huskamp HA, Keating NL. The impact of emerging safety and effectiveness evidence on the use of physician-administered drugs: the case of bevacizumab for breast cancer. Med Care. 2013;51(7):622-7. doi:10.1097/MLR.0b013e318290216f.

20. European Medicines Agency. 2013 annual report on EudraVigilance for the European Parliament, the Council and the Commission. 2014. http://www.ema.europa.eu/docs/en_GB/document_ library/Report/2014/04/WC500165780.pdf.

21. FDA. Reports received and reports entered into AERS by year. 2012. http://www.fda.gov/Drugs/GuidanceComplianceRegulatory Information/Surveillance/AdverseDrugEffects/ucm070434.htm. Accessed August 2014.
22. Hoffman KB, Dimbil M, Erdman CB, Tatonetti NP, Overstreet BM. The Weber effect and the United States Food and Drug Administration's Adverse Event Reporting System (FAERS): analysis of sixty-two drugs Approved from 2006 to 2010. Drug Saf Int J Med Toxicol Drug Exp. 2014. doi:10.1007/s40264-014-0150-2.

23. Staffa JA, Dal Pan GJ. Regulatory innovation in postmarketing risk assessment and management. Clin Pharmacol Ther. 2012;91(3):555-7. doi:10.1038/clpt.2011.289.

24. Harrington CA, Garcia AS, Sircar-Ramsewak F. Evaluation of adverse drug event information in US manufacturer labels. Curr Drug Saf. 2011;6(1):30-5.

25. Congress US. Food and Drug Administration Amendments Act of 2007 (110th Congress Public Law 85). 2007. http://www.gpo. gov/fdsys/pkg/PLAW-110publ85/html/PLAW-110publ85.htm. Accessed August 2014.

26. Cook DM, Gurugubelli RK, Bero LA. Risk management policy and black-box warnings: a qualitative analysis of US FDA proceedings. Drug Saf Int J Med Toxicol Drug Exp. 2009;32(11): 1057-66. doi:10.2165/11316670-000000000-00000.

27. Evans RW, Tepper SJ, Shapiro RE, Sun-Edelstein C, Tietjen GE. The FDA alert on serotonin syndrome with use of triptans combined with selective serotonin reuptake inhibitors or selective serotonin-norepinephrine reuptake inhibitors: American Headache Society position paper. Headache. 2010;50(6):1089-99. doi:10.1111/j.1526-4610.2010.01691.x.

28. Salinas GD, Robinson CO, Abdolrasulnia M. Primary care physician attitudes and perceptions of the impact of FDA-proposed REMS policy on prescription of extended-release and long-acting opioids. J Pain Res. 2012;5:363-9. doi:10.2147/jpr.s35798.

29. Zivin K, Pfeiffer PN, Bohnert AS, Ganoczy D, Blow FC, Nallamothu BK, et al. Evaluation of the FDA warning against prescribing citalopram at doses exceeding $40 \mathrm{mg}$. Am J Psychiatry. 2013;170(6):642-50. doi:10.1176/appi.ajp.2013.12030408.

30. Wilkinson JJ, Force RW, Cady PS. Impact of safety warnings on drug utilization: marketplace life span of cisapride and troglitazone. Pharmacotherapy. 2004;24(8):978-86.

31. Shatin D, Gardner JS, Stergachis A, Blough D, Graham D. Impact of mailed warning to prescribers on the co-prescription of tramadol and antidepressants. Pharmacoepidemiol Drug Saf. 2005;14(3):149-54. doi:10.1002/pds.961.

32. Barry CL, Martin A, Busch SH. ADHD medication use following FDA risk warnings. J Mental Health Policy Econ. 2012;15(3): 119-25.

33. Sclar DA, Robison LM, Castillo LV, Schmidt JM, Bowen KA, Oganov AM, et al. Concomitant use of triptan, and SSRI or SNRI after the US Food and Drug Administration alert on serotonin syndrome. Headache. 2012;52(2):198-203. doi:10.1111/j.15264610.2011.02067.x.

34. Woosley RL. Drug labeling revisions-guaranteed to fail? JAMA. 2000;284(23):3047-9.

35. Andrews E, Dombeck M. The role of scientific evidence of risks and benefits in determining risk management policies for medications. Pharmacoepidemiol Drug Saf. 2004;13(9):599-608. doi: $10.1002 /$ pds. 899 .

36. Goldman SA. Communication of medical product risk: how effective is effective enough? Drug Saf Int J Med Toxicol Drug Exp. 2004;27(8):519-34.

37. Nigrovic LE, Thompson KM. The Lyme vaccine: a cautionary tale. Epidemiol Infect. 2007;135(1):1-8. doi:10.1017/s0950268 806007096.

38. Motola D, Vargiu A, Leone R, Conforti A, Moretti U, Vaccheri $\mathrm{A}$, et al. Influence of regulatory measures on the rate of spontaneous adverse drug reaction reporting in Italy. Drug Saf Int J Med Toxicol Drug Exp. 2008;31(7):609-16.

39. O'Connor NR. FDA boxed warnings: how to prescribe drugs safely. Am Fam Physician. 2010;81(3):298-303. 
40. Smalley W, Shatin D, Wysowski DK, Gurwitz J, Andrade SE, Goodman M, et al. Contraindicated use of cisapride: impact of food and drug administration regulatory action. JAMA. 2000;284(23):3036-9.

41. Graham DJ, Drinkard CR, Shatin D, Tsong Y, Burgess MJ. Liver enzyme monitoring in patients treated with troglitazone. JAMA. 2001;286(7):831-3.

42. Willy ME, Manda B, Shatin D, Drinkard CR, Graham DJ. A study of compliance with FDA recommendations for pemoline (Cylert). J Am Acad Child Adolesc Psychiatry. 2002;41(7): 785-90.

43. Cluxton RJ Jr, Li Z, Heaton PC, Weiss SR, Zuckerman IH, Moomaw CJ, et al. Impact of regulatory labeling for troglitazone and rosiglitazone on hepatic enzyme monitoring compliance: findings from the state of Ohio medicaid program. Pharmacoepidemiol Drug Saf. 2005;14(1):1-9. doi:10.1002/pds.1048.

44. Wagner AK, Chan KA, Dashevsky I, Raebel MA, Andrade SE, Lafata JE, et al. FDA drug prescribing warnings: is the black box half empty or half full? Pharmacoepidemiol Drug Saf. 2006;15(6):369-86. doi:10.1002/pds.1193.

45. Gleason PP, Walters C, Heaton AH, Schafer JA. Telithromycin: the perils of hasty adoption and persistence of off-label prescribing. JMCP. 2007;13(5):420-5.

46. Morrato EH, Staffa JA. Effectiveness of risk management plans: a case study of pemoline using pharmacy claims data. Pharmacoepidemiol Drug Saf. 2007;16(1):104-12. doi:10.1002/pds.1279.

47. Nemeroff CB, Kalali A, Keller MB, Charney DS, Lenderts SE, Cascade EF, et al. Impact of publicity concerning pediatric suicidality data on physician practice patterns in the United States. Arch Gen Psychiatry. 2007;64(4):466-72. doi:10.1001/archpsyc. 64.4.466.

48. Morrato EH, Libby AM, Orton HD, Degruy FV 3rd, Brent DA, Allen R, et al. Frequency of provider contact after FDA advisory on risk of pediatric suicidality with SSRIs. Am J Psychiatry. 2008;165(1):42-50. doi:10.1176/appi.ajp.2007.07010205.

49. Olfson M, Marcus SC, Druss BG. Effects of Food and Drug Administration warnings on antidepressant use in a national sample. Arch Gen Psychiatry. 2008;65(1):94-101. doi:10.1001/ archgenpsychiatry.2007.5.

50. Starner CI, Schafer JA, Heaton AH, Gleason PP. Rosiglitazone and pioglitazone utilization from January 2007 through May 2008 associated with five risk-warning events. JMCP. 2008;14(6): 523-31.

51. Libby AM, Orton HD, Valuck RJ. Persisting decline in depression treatment after FDA warnings. Arch Gen Psychiatry. 2009;66(6):633-9. doi:10.1001/archgenpsychiatry.2009.46.

52. Hassanin H, Harbi A, Saif A, Davis J, Easa D, Harrigan R. Changes in antidepressant medications prescribing trends in children and adolescents in Hawaii following the FDA black box warning. Hawaii Med J. 2010;69(1):17-9.

53. Orrico KB, Lin JK, Wei A, Yue $\mathrm{H}$. Clinical consequences of disseminating the rosiglitazone FDA safety warning. Am J Manag Care. 2010;16(5):e111-6.

54. Shah ND, Montori VM, Krumholz HM, Tu K, Alexander GC, Jackevicius CA. Responding to an FDA warning: geographic variation in the use of rosiglitazone. New Engl J Med. 2010;363(22):2081-4. doi:10.1056/NEJMp1011042.

55. Valluri S, Zito JM, Safer DJ, Zuckerman IH, Mullins CD, Korelitz JJ. Impact of the 2004 Food and Drug Administration pediatric suicidality warning on antidepressant and psychotherapy treatment for new-onset depression. Med Care. 2010;48(11):947-54. doi:10. 1097/MLR.0b013e3181ef9d2b.

56. Hurren KM, Taylor TN, Jaber LA. Antidiabetic prescribing trends and predictors of thiazolidinedione discontinuation following the 2007 rosiglitazone safety alert. Diabetes Res Clin Pract. 2011;93(1):49-55. doi:10.1016/j.diabres.2011.02.035.
57. Shrank WH, Choudhry NK, Tong A, Myers J, Fischer MA, Swanton K, et al. Warnings without guidance: patient responses to an FDA warning about ezetimibe. Med Care. 2012;50(6): 479-84. doi:10.1097/MLR.0b013e31825517b6.

58. Davidson MH, Clark JA, Glass LM, Kanumalla A. Statin safety: an appraisal from the adverse event reporting system. Am J Cardiol. 2006;97(8a):32c-43c. doi:10.1016/j.amjcard.2005.12.008.

59. Garg V, Raisch DW, McKoy JM, Trifilio SM, Holbrook J, Edwards BJ, et al. Impact of United States Food and Drug Administration's boxed warnings on adverse drug reactions reporting rates and risk mitigation for multiple myeloma drugs. Exp Opin Drug Saf. 2013;12(3):299-307. doi:10.1517/14740338. 2013.780024

60. Pariente A, Gregoire F, Fourrier-Reglat A, Haramburu F, Moore $\mathrm{N}$. Impact of safety alerts on measures of disproportionality in spontaneous reporting databases: the notoriety bias. Drug Saf Int J Med Toxicol Drug Exp. 2007;30(10):891-8.

61. de Boissieu P, Kanagaratnam L, Abou Taam M, Roux MP, Drame M, Trenque T. Notoriety bias in a database of spontaneous reports: the example of osteonecrosis of the jaw under bisphosphonate therapy in the French national pharmacovigilance database. Pharmacoepidemiol Drug Saf. 2014;. doi:10.1002/pds. 3622.

62. De Bruin ML, van Puijenbroek EP, Egberts AC, Hoes AW, Leufkens HG. Non-sedating antihistamine drugs and cardiac arrhythmias: biased risk estimates from spontaneous reporting systems? Br J Clin Pharmacol. 2002;53(4):370-4.

63. Ben-Hamou M, Marshall NS, Grunstein RR, Saini B, Fois RA. Spontaneous adverse event reports associated with zolpidem in Australia 2001-2008. J Sleep Res. 2011;20(4):559-68. doi:10. 1111/j.1365-2869.2011.00919.x.

64. Raschi E, Piccinni C, Poluzzi E, Marchesini G, De Ponti F. The association of pancreatitis with antidiabetic drug use: gaining insight through the FDA pharmacovigilance database. Acta Diabetol. 2013;50(4):569-77. doi:10.1007/s00592-011-0340-7.

65. McAdams M, Staffa J, Dal Pan G. Estimating the extent of reporting to FDA: a case study of statin-associated rhabdomyolysis. Pharmacoepidemiol Drug Saf. 2008;17(3):229-39. doi:10. 1002/pds. 1535 .

66. Hoffman KB, Overstreet BM, Doraiswamy PM. A drug safety ePlatform for physicians, pharmacists and consumers based on post-marketing adverse events. Drugs Therapy Stud. 2013;3(e4).

67. RxNorm. National Library of Medicine. http://www.nlm.nih.gov/ research/umls/rxnorm/. Accessed August 2014.

68. FDA. Pharmacological Class: National Drug File Reference Terminology. 2013. http://www.fda.gov/ForIndustry/DataStandards/ StructuredProductLabeling/ucm162549.htm. Accessed August 2014.

69. MedDRA. Medical Dictionary for Regulatory Activities and the Maintenance and Support Services. 2013. http://www. meddramsso.com Accessed August 2014.

70. WHO. WHO Collaborating Centre for Drug Statistics Methodology. Guidelines for ATC classification and DDD assignment, 2013. http://www.whocc.no. Accessed August 2014.

71. FDA. MedWatch: The FDA Safety Information and Adverse Event Reporting Program. 2014. http://www.fda.gov/Safety/ MedWatch/. Accessed August 2014.

72. FDA. U.S. Food and Drug Administration. 2014. http://www.fda. gov/. Accessed August 2014.

73. Yong PL, Bigman C, Flynn DN, Mittermaier D, Long JA. Messages about black-box warnings: a comparative analysis of reports from the FDA and lay media in the US. Drug Saf Int J Med Toxicol Drug Exp. 2009;32(12):1147-57. doi:10.2165/ 11318960-000000000-00000.

74. Cassels A, Hughes MA, Cole C, Mintzes B, Lexchin J, McCormack JP. Drugs in the news: an analysis of Canadian newspaper 
coverage of new prescription drugs. CMAJ 2003;168(9): 1133-1137.

75. Center PR. Pew Internet \& American Life Project: Health Online. 2013. http://www.pewinternet.org/ /media/Files/Reports/2013/ PewInternetHealthOnlinereport.pdf. Accessed August 2014.

76. Moynihan R, Bero L, Ross-Degnan D, Henry D, Lee K, Watkins $\mathrm{J}$, et al. Coverage by the news media of the benefits and risks of medications. New Engl J Med. 2000;342(22):1645-50. doi:10. 1056/nejm200006013422206.

77. Barry CL, Busch SH. News coverage of FDA warnings on pediatric antidepressant use and suicidality. Pediatrics. 2010;125(1):88-95. doi:10.1542/peds.2009-0792.

78. Iaboli L, Caselli L, Filice A, Russi G, Belletti E. The unbearable lightness of health science reporting: a week examining Italian print media. PLoS One. 2010;5(3):e9829. doi:10.1371/journal. pone.0009829.
79. Weatherby LB, Nordstrom BL, Fife D, Walker AM. The impact of wording in "Dear doctor" letters and in black box labels. Clin Pharmacol Ther. 2002;72(6):735-42. doi:10.1067/mcp.2002. 129503.

80. Lasser KE, Seger DL, Yu DT, Karson AS, Fiskio JM, Seger AC, et al. Adherence to black box warnings for prescription medications in outpatients. Arch Intern Med. 2006;166(3):338-44. doi:10.1001/archinte.166.3.338.

81. Kurdyak PA, Juurlink DN, Mamdani MM. The effect of antidepressant warnings on prescribing trends in Ontario, Canada. Am J Public Health. 2007;97(4):750-4. doi:10.2105/ajph.2006.087262.

82. Matlock A, Allan N, Wills B, Kang C, Leikin JB. A continuing black hole? The FDA boxed warning: an appeal to improve its clinical utility. Clin Toxicol. 2011;49(6):443-7. doi:10.3109/ 15563650.2011 .564585 . 